(ㄹ)

CORPUS PUBLISHERS

\section{Current Research} in Emergency Medicine (CREM)

ISSN: 2832-5699

Volume 2, Issue 1, 2022

Article Information

Received date : 14 December, 2021

Published date: 11 February, 2022

*Corresponding author

Marion-Vincent Mempin, MSc, MD, New York Presbyterian Queens, Envision Healthcare, Department of Emergency Medicine, Flushing, NY, USA.

Key Words

Pain; Intraluminal Hemorrhage; Hematuria; Urine Leak; Urogenital Trauma

DOI: 10.54026/CREM/1019

Distributed under: Creative Commons CC-BY 4.0

\title{
Case Report: Traumatic Abdominal Pain and Hematuria
}

\section{Brian Smith, Mario-Vincent Mempin*}

Department of Emergency Medicine, New York Presbyterian Queens, Envision Healthcare, Flushing, New York, USA

\section{Abstract}

We report a case of a 56-year-old male presented with diffuse abdominal pain after falling 2 feet off a ladder. He underwent an in-depth laboratory workup (together with an automated urine microscopy) A CT cystogram was obtained and negative for bladder perforation. He was then brought to Interventional Radiology for angiography of the left kidney. No active extravasation was seen and the patient was admitted to the surgical intensive care unit. Urology recommended continuous bladder irrigation for removal of clot. A CT urogram showed urine leak from left kidney and was treated with a left ureteral stent. The affected person remained hemodynamically solid and became discharged home.

Chief Complaint: Abdominal Pain

Background

A 56 year-old male with no past medical history presented with diffuse abdominal pain after falling 2 feet off a ladder. The patient denied any head trauma or loss of consciousness. The patient complained of difficulty urinating and lightheadedness after the fall. His initial vital signs were significant for hypotension to $70 \mathrm{~s} / 40 \mathrm{~s}$.

Pertinent Physical Exam

General : AAOx3, pale, diaphoretic

CVS : RRR

Lungs : CTA b/l

Abd : Diffusely tender to palpation with guarding, nondistended. Left flank tenderness to percussion

GU : Blood at urethral meatus

Question

What is your diagnosis based on eFAST (top) and CT imaging (bottom)?

Answer

eFAST showed free fluid in the LUQ (top right) and a large intraluminal urinary bladder hematoma (top left). CT images show a grade 5 laceration of the left kidney extending to the renal hilum and collecting system (bottom right) and a distended bladder with intraluminal hemorrhage (bottom left).
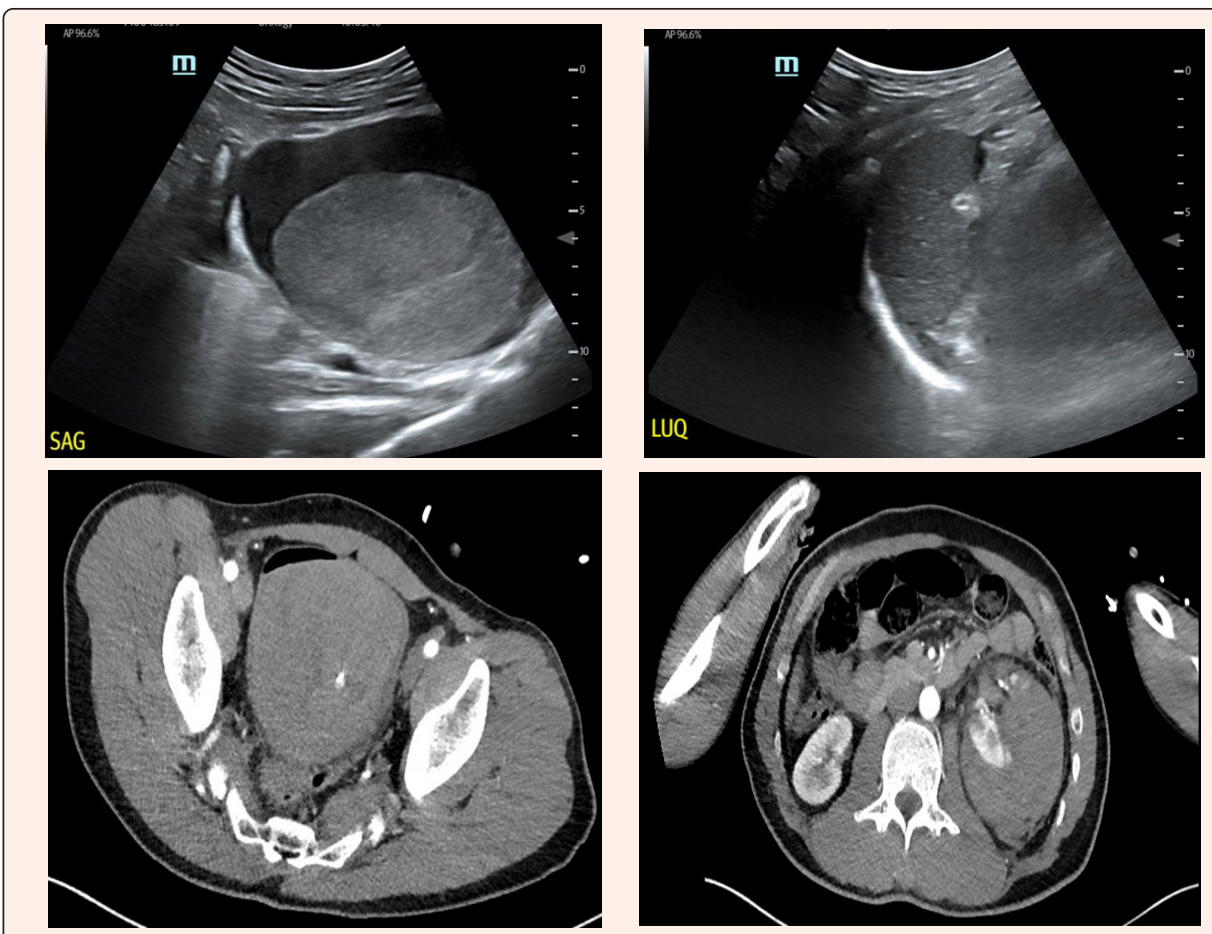


\section{Case Discussion}

Our massive transfusion protocol was activated and vascular access was obtained with bilateral $18 \mathrm{G}$ peripheral IVs, a femoral cordis, and a radial arterial line. The patien also received 1g TXA. A foley catheter was placed with a large volume of blood and clots evacuated. His hemodynamic status improved after these resuscitative measures. A CT cystogram was obtained and negative for bladder perforation. He was then brought to Interventional Radiology for angiography of the left kidney. No active extravasation was seen and the patient was admitted to the surgical intensive care unit. Urology recommended continuous bladder irrigation for removal of clot. On HD 2, repeat imaging showed an extensive left renal laceration with large perinephric hematoma. A CT urogram showed urine leak from left kidney and was treated with a left ureteral stent. The patient remained hemodynamically stable and was discharged home on hospital day 5 with foley catheter in place and instructions to follow up with urology in 1 week.

\section{Clinical Pearls}

1. Renal and urogenital injuries occur in $10-20 \%$ of abdominal traumas in adults.

2. While eFAST is a rapid and effective tool for detecting intra-abdominal free fluid, it has a low sensitivity and specificity in identifying traumatic kidney injuries.
3. For patients with hematuria and hypotension in the setting of blunt trauma, contrast-enhanced CT scan with delayed urographic phase is gold-standard in identifying kidney injuries.

4. Retrograde cystoscopy should be performed in hemodynamically stable patients with suspected bladder injury.

5. Management of urogenital traumas should be multidisciplinary- including emergency physicians, trauma surgeons, urologists, interventional radiologists, and ICU physicians.

\section{Conflicts of Interest: None}

\section{Financial Support: None}

\section{References}

1. Federico C, Moore EE, Kluger Y, Biffl W, Leppaniemi A, et al. (2019) Kidney and Uro-Trauma: WSES-AAST Guidelines. World J Emerg Surg 14: 54. 